\title{
The first year of Sars-Cov-2: Which mutations spread rapidly around the word? Minireview
}

\author{
Weimer LE ${ }^{1 *}$, Cattari G ${ }^{2}$, Fanales-Belasio $E^{3}$, Binelli $A^{1}$, Poddighe AF ${ }^{2}$ and Sensi $F^{2}$ \\ ${ }^{1}$ National Center for Global Health, Istituto Superiore di Sanità, Rome, Italy \\ ${ }^{2}$ SSD Long-term care-ASSL Sassari-ATS Sardinia, Italy \\ ${ }^{3}$ Department of Infectious Diseases, MIPI, Istituto Superiore di Sanità, Rome, Italy
}

\begin{abstract}
Severe acute respiratory syndrome coronavirus 2 (SARS-CoV-2) has caused a worldwide crisis with profound effects on both public health and the economy. In order to combat the Sars-Cov-2 pandemic, research groups have shared viral genome sequence data through the Global Initiative on Sharing All Influenza Data (GISAID).

SARS-CoV-2 is an RNA virus, which is a family with significant adaptive evolution).

Worldwide research groups are generating and sharing SARS-CoV-2 proteome sequence data in a rapid fashion as a global effort to combat the COVID-19 pandemic. The rapid spread of an alternative variant of coronavirus has been blamed for the introduction of strict tier four mixing rules for many people, strict restrictions on mixing at Christmas in England, Scotland and Wales, and other countries placing the United Kingdom on a travel ban.

So how has it gone from being non-existent to the foremost common sort of the virus in parts of England during a matter of months?

Now that vaccines have been developed and are being deployed to address the COVID-19 pandemic, a major concern is the emergence of mutations in severe acute respiratory syndrome coronavirus 2 (SARS-CoV-2) that confer immune escape or enhanced fitness. As such, it is important to assess how rapidly SARS-CoV-2 proteins are mutating. While it is currently unclear if these mutations benefit the virus, their continued surveillance and the detection of new proteome variants are likely to illuminate key aspects of viral function.
\end{abstract}

The government's advisers on new infections now say they need "high" confidence that it's more ready to transmit than other variants.

\section{Introduction}

Severe acute respiratory syndrome coronavirus 2 (SARS-CoV-2) has caused a worldwide crisis with profound effects on both public health and the economy. In order to combat the Sars-Cov- 2 pandemic, research groups have shared viral genome sequence data through the Global Initiative on Sharing All Influenza Data (GISAID) [1].

SARS-CoV-2 is an RNA virus, which is a family with significant adaptive evolution).

Worldwide research groups are generating and sharing SARS$\mathrm{CoV}-2$ proteome sequence data in a rapid fashion as a global effort to combat the COVID-19 pandemic.

Now that vaccines have been developed and are being deployed to address the COVID-19 pandemic, a major concern is the emergence of mutations in severe acute respiratory syndrome coronavirus 2 (SARSCoV-2) that confer immune escape or enhanced fitness. As such, it is important to assess how rapidly SARS-CoV-2 proteins are mutating . While it is currently unclear if these mutations benefit the virus, their continued surveillance and the detection of new proteome variants are likely to illuminate key aspects of viral function [2].

\section{Mini review}

\section{The temporal emergence of proteome mutations}

As coronaviruses have high adaptive evolution, we expect that SARS-CoV-2 presents significant temporal variations. Some factors can condition the different viral variants. Growing evidence indicates that climate and seasonal effects, including temperature, humidity, sunlight, and people's habits, can contribute to the expansion of the virus [3]. Country-specific factors, such as demography, cultural practices, social interventions, travel restrictions, quarantine policies, health care capacity, and reporting and tracking mechanisms, can also alter viral expansion and variation.

The first new strain was discovered by Public Health England's genomic surveillance. The agency notified the United Kingdom government on 18 December on the seriousness of the new strain, and therefore the UK submitted its findings to the planet Health Organization an equivalent day.

The new UK variant is believed to possess first emerged in southeast England in September. It has since become the dominant sort of the virus within the area. It has also been found in Denmark, Netherlands, Belgium and now around the wordwide.

The new strain may be a derivative of the D614G mutation, which appeared independently in multiple locations. However, just

${ }^{\star}$ Correspondence to: Liliana Elena Weimer, National Center for Global Health, Istituto Superiore di Sanità Rome, Italy, E-mail: liliana.weimer@iss.it

Key words: new strains, mutation, travel restrictions, vaccination

Received: February 16, 2021; Accepted: February 22, 2021; Published: February 25,2021 
one lineage of that mutation spread rapidly across the world, driven by human behaviour, consistent with virologists, and is now found in nearly all sequences [4].

Three things are coming together that mean it's attracting attention:

- It is rapidly replacing other versions of the virus

- It has mutations that affect part of the virus likely to be important

- Some of those mutations have already been shown in the lab to increase the ability of the virus to infect cells All of those close to create a case for an epidemic which will spread more easily .

\section{Have the new mutations spread around the world?}

It is thought the variant either emerged during a patient within the UK or has been imported from a rustic with a lower ability to watch coronavirus mutations.

The variant are often found across the United Kingdom, except Northern Ireland, but it's heavily concentrated in London, the South East and eastern England. Cases elsewhere within the country don't seem to possess began.

Data from Nextstrain, which has been monitoring the genetic codes of the viral samples round the world, suggest cases in Denmark and Australia have come from the United Kingdom. The Netherlands has also reported cases .

\section{How many times has the Sars-CoV-2 virus mutated?}

More mutations are being discovered every day .The scientists have noted two distinct set of mutations that are the most frequent. within the virus that causes Covid-19, the H69/V70 deletion, and therefore the D614G, both of which affects the spike proteins, which helps the virus to bind to the human cell, and infect it. It is this ability that vaccines seek to focus on and weaken. The H69- 70 deletion affects antibody susceptibility, and has been seen 3 times so far- in Danish minks, in Britain, and during a patient who was rendered much less responsive to convalescent plasma therapy.

The virus was first reported in Wuhan and since then, it's said to possess mutated repeatedly, most of which have gone unnoticed. The $\mathrm{BBC}$ reported that if the strain that's infecting people now's compared to the primary one that spread in Wuhan a complete of 25 mutations are going to be visible, that's a little over two mutations a month. As the physical body gets better at identifying and fighting it, the virus too is predicted to fight back, by trying to evade the body's system.

The D614G is that the first known mutation that's suspected to possess occurred in Eastern China in January and later spread through NY City and Europe. Within a couple of months of the pandemic this particular variant was found everywhere the planet, because it had successfully replaced its predecessor, which had spread from Wuhan.

\section{Are there other variants in the world?}

There are another new variants. One sequenced for the first time in Manaos ,Brazil , the second variant in South Africa and the third variant in Japan.Currently this variants has been observed globally (Figure 1).

Viruses, in particular RNA viruses such as coronavirus, constantly evolve through mutations and while most will not have a significant

\section{Mutation of SARS-CoV-2: current variants of concern \\ 8 February 2021}

Mutations of SARS-CoV-2 that cause COVID-19 have been observed globally.

Viruses, in particular RNA viruses such as coronaviruses, constantly evolve through mutations, and while most will not have a significant impact, some mutations may provide the virus with a selective advantage such as increased transmissibility. Such mutations are cause for concern and need to be monitored closely.

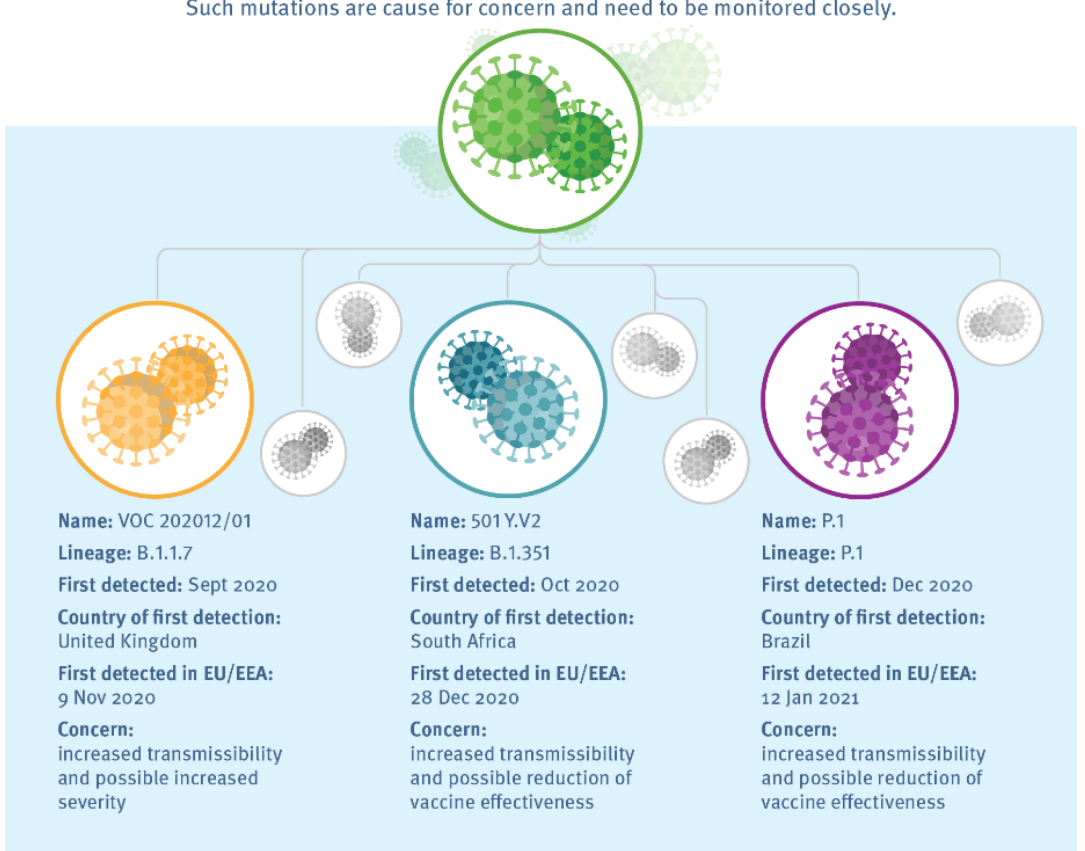

Figure 1. European Center for Disease Prevention and Control and Agency of European Union. Infografic 8 Feb 2021.Mutation of Sars-Cov-2-current and variants of concern 
impact, some mutations may provide the virus with a selective advantage such as increased transmissibility. Such mutations are cause for concern and need to be monitored closely [5].

\section{Will the variants be immune to current vaccine candidates?}

The possibility that the new variants are going to be immune to existing vaccine candidates is low, but not "inexistent".

Other researchers also say the mutations aren't expected to be immune to existing vaccine candidates, which can still protect against them. Unlike influenza viruses, the coronavirus evolves and mutates at a way slower rate.

However, as more and more people get vaccinated, researchers expect the virus to evolve mutations which will help make it immune to vaccines within the future.

Many countries around the world are restricting travel from Britain and elsewhere amid concerns about new strains of the coronavirus. The restrictions focus mainly on Britain, which has imposed strict new lockdown measures because of what it described as the unusually rapid spread of a new strain there. A few other European countries have confirmed cases of the virus .

\section{Conclusion}

The functional mean and evolutionary importance of most of the SARS-CoV-2 mutations are still being investigated.

\section{Conflicts of interest}

The authors do not have a commercial or other association that might pose a conflict of interest.

\section{Financial support}

None.

\section{References}

1. Vilar S, Isom D (2021) One Year of SARS-CoV-2: How much has the virus changed? Biology 10: 91.

2. Islam MR, Hoque MN, Rahman MS, Rubayet Ul Alam ASM, et al. (2020) Genomewide analysis of SARS-CoV-2 virus strains circulating worldwide implicates heterogeneity. Sci Rep 10: 14004. [Crossref]

3. Merow C, Urban MC (2020) Seasonality and uncertainty in global COVID-19 growth rates. Proc Natl Acad Sci USA 117: 27456-27464. [Crossref]

4. Swarna A (2020) Mini review - New emerging COVID strain. J Intensive Crit Care 6: 25.

5. https://www.ecdc.europa.eu/en/publications-data/covid-19-infographic-mutationscurrent-variants-concern

Copyright: $\odot 2021$ Weimer LE. This is an open-access article distributed under the terms of the Creative Commons Attribution License, which permits unrestricted use, distribution, and reproduction in any medium, provided the original author and source are credited. 